\title{
Editorial
}

\section{Synoptic reporting in pathology: maximum sense in minimal time}

\author{
Indranil Chakrabarti ${ }^{1, *}$ \\ ${ }^{1}$ Dept. of Pathology, North Bengal Medical College, Darjeeling, West Bengal, India
}

\section{A R T I C L E I N F O}

Article history:

Received 15-05-2020

Accepted 22-05-2020

Available online 11-06-2020
(C) 2020 Published by Innovative Publication. This is an open access article under the CC BY-NC license (https://creativecommons.org/licenses/by-nc/4.0/)
A pathological report is a precious document and is more than data to the patient. However, the reports are mostly presented in a narrative way and important information may be overlooked. The production of the report may also be inadequate as comments on important diagnostic and prognostic parameters may be missed. This causes a great deal of difficulty and hence dissatisfaction among the treating physicians. The requirement of completeness of reporting and generation of a standardized report is more importantly felt in oncologic pathology. As we move more and more towards structured and objective evaluation of all sort of data, a sincere need is felt to produce synoptic reports in pathology. In this direction, College of American pathologists' (CAP) electronic cancer protocols (https://www.cap.org/protocols-and-guide lines/cancer-reporting-tools/cancer-protocol-templates) and California Cancer registry (https://www.ccrcal.org/learn-ab out-ccr/about-cancer-registries) have long been promoting structured synoptic reporting for cancer patients. The CAP accreditation of laboratories also requires adherence to such protocols (LAP- Laboratory Accreditation Program). These electronic checklists are largely popular among pathologists who prefer to generate a more complete oncopathological report and also among oncologists as they receive a complete report and have to spend much less effort and time to extract all the valuable and necessary information. More recently, an International Collaboration

\footnotetext{
* Corresponding author.

E-mail address: drinch@ rediffmail.com (I. Chakrabarti).
}

on Cancer Reporting (ICCR) founded by major pathology organizations of the world, have published internationally standardized and evidence based datasets for the cancer reporting. ${ }^{1}$ The datasets are in line with the latest WHO classification of tumors and some are in the process of development with the aim to present the latest in cancer diagnostics. Both CAP and ICCR reporting formats can be downloaded, printed and filled up manually. The responses can be marked as present/not identified etc. The explanatory notes at the end of each format are an invaluable source of latest information. . CAP also has provision of CAP eFRM software that can be incorporated in the laboratory information system (LIS) making cancer reporting much more convenient, comprehensive, complete and consistent. In February, 2020, CAP has updated the protocols and has released 47 revised cancer protocols and 1 new adult autopsy reporting format. ${ }^{2}$

The word Synoptic comes from the Greek word "Synopsis" meaning overview ${ }^{3}$ as it provides the best possible overview of the submitted specimen in a structured manner. Though mostly used in cancer related reporting, synoptic reporting is also making inroads into non-neoplastic pathology reports. Apart from providing complete, accurate, standardized reports, this form of data representation can be easily fed, stored and searched by computers for sorting, and analyzing the individual data elements (database mapping). This can thus help immensely in data exchange, analysis, research and maintaining cancer registries. The trends and patterns can be studied with much ease and variables and 
outcomes can be measured, matched and maybe predicted.

There are 6 tiers of data structuring as per Ellis and Srigley 4,5

Level 1: Narrative report (no defined content)

Level 2: Narrative report with standardized content (e.g., by using a checklist for dictation)

Level 3: Synoptic report (adds a specific format, but not necessarily any underlying software Implementation)

Level 4: Synoptic report with electronic reporting tools

Level 5: Standardised structured report with underlying database structure

Level 6: Standardised structured report with binding terminology in order to facilitate data Exchange.

A web based synoptic reporting using standardized softwares and linking with SNOMED-CT and LOINC ${ }^{3,6}$ will ultimately result in integration of standardized data worldwide and may revolutionize the patient care and research in the coming years. But more importantly, as of today, achieving a Level 3 report using available international protocols will require no extra effort and is very much recommended. However, the copyright issues must be checked from the CAP website to avoid any legal issues.

In the beginning the task of filling up the checklists may seem cumbersome and time taking. Some may even feel a lack of flexibility in reporting as this form of reporting based on eCC (electronic Cancer checklists) leaves very little to ambiguity. However, the use of generating a complete, wellstructured report largely outweighs these shortcomings.

To conclude, synoptic reporting will not only be beneficial for data analytics but for the generation of accurate, complete, standardized, structured information of the patient sample from which the most valuable information can be extracted with rapidity, ease and accuracy. Web based synoptic reporting platforms appear to hold promise in near future.

\section{Source of Funding}

None.

\section{Conflict of Interest}

None.

\section{References}

1. http://www.iccr-cancer.org.

2. https://www.cap.org/protocols-and-guidelines/cancer-reporting-tools/c ancer-protocol-templates.

3. Hewer E. The Oncologist's Guide to Synoptic Reporting: A Primer. Oncol. 2019;7:1-7.

4. Ellis DW. Surgical pathology reporting at the crossroads: beyond synoptic reporting. Pathol. 2011;43(5):404-9.

5. Ellis DW, Srigley J. Does standardised structured reporting contribute to quality in diagnostic pathology? The importance of evidence-based datasets. Virchows Arch. 2016;468(1):51-9.

6. Campbell WS, Karlsson D, Vreeman DJ, Lazenby AJ, Talmon GA, Campbell JR, et al. A computable pathology report for precision medicine: extending an observables ontology unifying SNOMED CT and LOINC. J Am Med Inform Assoc. 2018;25(3):259-66.

\section{Author biography}

Indranil Chakrabarti Associate Professor

Cite this article: Chakrabarti I. Synoptic reporting in pathology: maximum sense in minimal time. IP Arch Cytol Histopathology Res 2020;5(2):109-110. 